\title{
2 Globales Spanisch als Effekt von Language Making: Wirkungen von Spracheinstellungen und -ideologien
}

Was ist das Spanische? Jede Sprache ist in erster Linie eine „idea in the mind“, wie es Milroy (2001: 543) für Standardsprachen ausdrückt. Dies gilt nicht nur für die Repräsentation, die wir von festgelegten und weithin akzeptierten sprachlichen Normen einer Standardsprache haben, sondern es gilt auch für das Gesamtbild einer ,Einzelsprache‘, selbst wenn diese nicht standardisiert oder kodifiziert ist. Was wir als eine Sprache wahrnehmen, basiert auf einer kollektiven Konstruktion dessen, was wir uns unter dieser Sprache vorstellen. Es ist die Oberfläche eines stets andauernden Prozesses von Language Making. ${ }^{5}$

Mit dem Konzept Language Making ist gemeint, dass bewusst oder unbewusst durch menschliches Handeln imaginierte bzw. konstruierte Einheiten entstehen, die wir als Einzelsprachen erfassen. Diese Sprachen werden als abgrenzbar konzeptualisiert, in der Regel erhalten sie Namen oder Labels und sie werden mit Normen belegt. Eine Rolle spielen dabei häufig strukturelle Normen wie Orthographie, eine präskriptive Grammatik oder ein kodifizierter Wortschatz, aber auch bei nicht formal kodifizierten Sprachen bestehen funktionale Normen wie bestimmte Gebrauchskonventionen oder -vorschriften, soziale Konnotationen des Sprachgebrauchs und weithin akzeptierte bzw. abgelehnte Verwendungsdomänen für Sprachformen, die als Teil der benannten Einheit ,Einzelsprache“ gesehen werden. Ausgeschlossen werden dabei Erscheinungsformen, die als nicht dem vermeintlich abgegrenzten Normsystem zugehörig angesehen werden. Makoni und Pennycook (2005) sprechen in diesem Zusammenhang von „disinventing“ und „(re)constitution“ von Sprachen. Language Making ist keineswegs gleichbedeutend mit Standardisierung oder gar Sprachplanung, denn Verwendungsnormen und -konventionen, Abgrenzungen und Labels können auch auf Sprachformen angewendet werden, die nicht strukturell standardisiert oder beispielsweise verschriftlicht sind.

Getragen wird das Language Making von Spracheinstellungen und sprachideologischen Grundlagen. Dragojevic (2017: 3) fasst den Begriff Spracheinstellung recht knapp als „evaluative reactions to different language varieties. “ Im vorliegenden Fall geht es also darum, wie Menschen das Spanische bewerten,

5 Eine ausführlichere Beschreibung des Konzepts Language Making ist zum Zeitpunkt des Erscheinens dieses Buches in der Vorbereitung, vgl. Krämer/Vogl/Kolehmainen (in Vorb.). 
insbesondere im Kontext ihrer eigenen Lernabsichten. Die wertenden Reaktionen lassen sich weiter aufgliedern, etwa als „sets of beliefs about language articulated by the users as a rationalization or justification of perceived language structure and use“ (Silverstein 1979: 193). Damit wird deutlicher, dass die Wertung an innersprachliche Eigenschaften geknüpft werden kann, etwa ästhetische Wahrnehmungen der Aussprache oder Konnotationen des Wortschatzes, aber auch an die Sprachnutzung an sich. Letzteres ist beispielsweise häufig der Fall, wenn die übermäßige Nutzung des Englischen in Domänen wie der Werbung oder der Wirtschaftskommunikation beklagt wird. Spracheinstellungen sind zunächst Haltungen der Einzelperson gegenüber einer sprachlichen Erscheinungsform:

Unter Spracheinstellungen verstehen wir zu Haltungen verfestigte Meinungen eines Individuums zu Sprache und Sprechern, die mit den jeweiligen individuellen sprachlichen und allgemeinen (tatsächlichen oder vermeintlichen, stabilen oder vagen) Wissensbeständen in Beziehung stehen; als psychische Dispositionen können sie entscheidungsund handlungsleitend sein; sie können den Sprechern in weiten Teilen unbewusst sein; und sie sind individuell unterschiedlich scharf konturiert.

(Adler / Plewnia 2018: 63)

Die psychischen Dispositionen, die hier zunächst nur angedeutet werden, lassen sich nach Dragojevic (2017: 3) als eine „tripartite view of attitudes“ einteilen in kognitive, affektive und Verhaltenskomponenten. ${ }^{6}$ Die Einstellungen von einzelnen Personen fügen sich zusammen zu übergeordneten Ideologien, die von vielen Individuen geteilt werden. Man kann Sprachideologien deshalb als eine Art ,shared subjectivity“ sehen, als subjektive Blicke auf Sprache, die von vielen Individuen geteilt werden. Woolard (2007: 130) beschreibt die Wirkungsweise von Ideologien wie folgt: „La ideología no refleja sino que refracta las relaciones sociales que la generan y que a la vez son organizadas por ella.“ Die Existenz von Einzelsprachen wird demnach aus sozialen Verhältnissen generiert; sie ist damit in gewissem Sinne virtuell, aber dennoch in der Wahrnehmung der Sprachgemeinschaft und in ihrer Sprechpraxis sehr real. Auch Kroskrity (2004: 497, 505) hebt zu Recht hervor, dass man Spracheinstellungen und -ideologien nicht ohne Weiteres als bewusste Größen annehmen kann: Sie bleiben oft implizit und unhinterfragt. Ziel der Forschung ist es daher, sie an die Oberfläche zu holen. Da der Entscheidung für das Erlernen einer bestimmten Sprache zumeist ein Reflexionsprozess vorausgeht, kann man in diesem Zusammenhang davon ausgehen, dass die Spracheinstellungen relativ leicht zugänglich sind. Anders als in Situationen, in denen beispielsweise gesellschaftliche Tabus betroffen sind, dürfte im

6 Eine angepasste Einteilung dieser drei Komponenten leitet die Gliederung der Befragungsstudien, die in Kapitel 6 ausführlicher vorgestellt wird. 
vorliegenden Fall also das Bewusstsein für die eigenen Haltungen zur Sprache und auch die Bereitschaft, darüber Auskunft zu geben, recht hoch sein.

Mit der Wahl des Spanischen als Fremdsprache schließen sich die Lernenden einer Gemeinschaft an, die mit ihren Praktiken, ihren Vorstellungen und Einstellungen das Spanische formt. Sie werden damit Teil einer Diskursgemeinschaft, die basierend auf ihren Sprachideologien mitentscheiden, was das Spanische ist und welche Bedeutung es für sie hat (Valle 2007a: 17, Paffey 2012: 80-103, Kroskrity 2004: 501). Während Irvine/Gal (2000: 77) in der Soziolinguistik einen ,shift of attention from linguistic communities to linguistic boundaries“ beobachten, so hat das Konzept des Language Making das Potenzial, beides zusammenzuführen. Die in den späteren Kapiteln vorgestellten Befragungen testen kleine Diskurselemente, die auf Spracheinstellungen rückschließen lassen und damit ein Stück weit offenlegen, wie die Lernenden sich eine Vorstellung des Spanischen bilden.

Zentral für Language Making ist, dass der Prozess nie abgeschlossen ist, ebenso wie auch Spracheinstellungen und -ideologien wandelbar sind. Eine Sprache als Sprache wird stets wieder neu konstruiert bzw. ihr Status als Sprache bestätigt und gefestigt, und zwar durch das Sprechen bzw. Schreiben in der Sprache und über die Sprache, mit Bourdieu gesprochen: „[L]es ,langues“ n’existent qu’à l'état pratique, c'est-à-dire sous la forme d'habitus linguistiques au moins partiellement orchestrés et de productions orales de ces habitus“ (Bourdieu 2001 [1982]: 72). Auch wenn Bourdieu in erster Linie seinen linguistischen Markt innerhalb einer Sprachgemeinschaft ansiedelt und darin die Sanktionierung bzw. Legitimität bestimmter Formen in den Blick nimmt, lassen sich seine Überlegungen ohne Weiteres auf die Verhandlung von symbolischen und materiellen Werten unterschiedlicher Gesamtsprachen und deren Konstruktion übertragen. ${ }^{7}$

(Teil-)Prozesse des Language Making können durchaus auch gezielt und gesteuert stattfinden, beispielsweise beim Ausbau von Minderheitensprachen oder bei Standardisierungsprozessen. Bourdieu (2001 [1982]: 77) spricht in diesem Zusammenhang von einer „fabrication de la langue“, die er in einen direkten Zusammenhang mit der Herausbildung der europäischen Nationalstaaten stellt. Der politische Kontext spielt dabei eine zentrale Rolle, wie es Valle (2013: 18) in seinem Konzept des Language Making in Bezug auf die Sprachgeschichte des Spanischen hervorhebt: „Spanish is approached as a discursively constructed political artifact that, as such, contains traces of the society in which it is produced and of the

7 Vgl. umfassend Bourdieu (2001 [1982]: 100-107), zur Übertragbarkeit aber auch Bourdieus (2001 [1982]: 87) eigene Überlegungen zur Verteidigung des Lateinischen, bei dem in der Regel intrinsische Werte der Sprache genannt werden, letztendlich aber in erster Linie ein Markt beibehalten werden soll, auf dem bestimmte Zugangshürden zum Lateinischen als Mittel der Verknappung dienen. 
discursive traditions that are involved - and often even invoked - in its creation.“ Trotz der vermeintlich fixierenden Wirkung eines Standards und politischer bzw. rechtlicher Regelungen bleibt auch bei Standardsprachen und offiziell anerkannten Sprachen der Vorgang des Language Making stets unvollkommen: Innerhalb des Standards ist immer zumindest ein kleiner Rest von Variation erhalten. Plurizentrische Sprachen sind besonders einleuchtende Beispiele (zum Konzept der Plurizentrik vgl. grundlegend Clyne 1992, 2004; Auer 2014: 18-20; speziell für das Spanische die Beiträge in Greußlich/Lebsanft, Hg. 2020). Zugleich ist immer wieder auch die Zugehörigkeit bestimmter Formen zum Standard bzw. ihr Ausschluss davon in der Sprachgemeinschaft umstritten. Die Reichweite des Standards, seine soziale Funktion, und das Verhältnis zu anderen, als Einheiten gefassten Sprachen, bleibt ebenso wandelbar.

Das Spanische als Einzelsprache ist daher in erster Linie kein abgeschlossenes historisch-strukturelles System mit seiner Grammatik und seinem Wortschatz, sondern es ist eine durchaus variable Praxis mit einem gemeinsamen Bestand an Formen, die als Erscheinung des Language Making zusammengehalten wird von einem Set an Vorstellungen, Funktionen und Zuschreibungen, die mit dem Label Spanisch belegt sind.

Wie Kroskrity (2004: 497) und Irvine (1989: 255) unterstreichen, sind Spracheinstellungen und -ideologien stets in einen lokalen Kontext eingebunden, der kulturelle, politische, historische und ökonomische Spezifika aufweist. Besonders bei einer praktisch weltumspannend präsenten Sprache wie dem Spanischen liegt es auf der Hand, dass die sprachbezogenen Wertungen nicht überall identisch sein werden. In verschiedenen Kontexten oder Gesellschaften können also auch im Rahmen des Language Making die Vorstellungen und Zuschreibungen durchaus unterschiedlich ausfallen: „El lenguaje mismo [. . .] exige ser definido como fenómeno ideológico-discursivo, es decir, como entidad dinámica en constante relación dialógica con el contexto“ (Valle 2007a: 14; vgl. auch Valle 2007c: 82). Global Spanish setzt sich zusammen aus zahlreichen Elementen, die bisweilen auch widersprüchlich sind: In Spanien spielt das castellano als dominante Sprachform eine herausragende Rolle, es ist allerdings in vielen Regionen des Landes zugleich auch stark politisch konnotiert und damit ambivalenten Einstellungen unterworfen. In Lateinamerika erfüllte das Spanische eine zentrale Funktion in den Prozessen des postkolonialen nation building, obwohl (und teils auch weil) es die Sprache der ehemaligen Kolonialmacht war. In den USA wiederum ist die spanische Sprachgemeinschaft trotz - oder gerade wegen - ihrer millionenstarken Präsenz einer äußerst wirksamen Marginalisierung und Ablehnung durch die anglophone Mehrheit ausgesetzt (Fuller 2013: 13-18, García 2011, Mar-Molinero 2010: 164). In vielen anderen Teilen des ,globalen Nordens‘ genießt das Spanische hingegen einen gänzlich anderen Status, der selbstverständlich auch in den 
USA verbreitet ist und dort den Abwertungen des Spanischen entgegensteht. ${ }^{8}$ Spanisch ist in diesen Fällen Ausdrucksmittel eines Lebensgefühls und -stils, der assoziiert wird mit Ungezwungenheit und Lockerheit, mit bestimmten Musikkulturen, mit Freizeit und dem Ausdruck von Lebensfreude, gewissermaßen als Gegenpol zur strengen Rationalität der ökonomisierten ,nördlichen“ Realität (Mar-Molinero/Paffey 2011: 759, Cepeda 2000, Schneider 2014). Neben dem Spanischen als Nationalsymbol und dem Spanischen als migrantisch markierte Minderheitensprache besteht somit ein ,drittes' Spanisch, nämlich das einer globalen Praxis, die allerdings mit den anderen beiden Vorstellungen eng verflochten ist.

Valle (2007a: 20) betont, dass neben dem politisch-sozialen und historischen Kontext bei der Betrachtung von Sprachideologien auch die ,función naturalizadora“, also die Erschaffung außersprachlicher Realitäten und Wirkungen durch die Ideologien, und die ,institucionalidad“, also die institutionell gefestigte Verstetigung von Ideologien, mit der Machtverhältnisse gefestigt werden. Institutionen und deren Wirkungswege schaffen ein Zusammengehörigkeitsgefühl, das sich unter anderem durch „Loyalität“ gegenüber den Sprachnormen ausdrückt, wie sie vor allem durch formale Bildungssysteme vermittelt werden (Valle 2007b: 41).

Für die Situation in Berlin bedeutet das: Im Mittelpunkt der Betrachtung stehen Lehreinrichtungen. Diese haben einen besonders starken Einfluss auf die Verstetigung von Sprachideologien, sie wirken am Language Making entscheidend mit und sind damit zentrale Institutionen für den Prozess. Beteiligt sind aber nicht nur die Institutionen als solche durch ihre herausgehobene gesellschaftliche Stellung vielmehr stehen Lehrende und Lernende miteinander in Kontakt, und auch die Lernenden untereinander. In diesem Verhältnis konzentriert sich der metasprachliche Diskurs, der entscheidende Spracheinstellungen abbildet. Wer in Berlin Spanisch lernt, ist damit Teil einer relativ spezifischen Gruppe an einem bestimmten Ort zu einer bestimmten Zeit, in einer bestimmten Umgebung, die selbst jedoch Teil eines viel größeren Diskursuniversums ist, in dem das Konzept Spanisch verhandelt wird.

Das entstehende Gesamtbild des ,globalen Spanischen“ prägt die Motivationen zum Lernen und die Erwartungen, die an die Beherrschung der Sprache geknüpft werden. Sie haben Wirkungen bis hinein ins klassische Bildungswesen, wo das Spanische als Fremdsprache inzwischen fest verankert ist (vgl. hierzu ausführlich Kapitel 5). Noch ist weltweit das Spanische eine Sprache, die häufiger

8 Vgl. die Beobachtung von Pomerantz (2002: 276): „The value attached to Spanish by foreign language learners in elite academic institutions often stands in marked contrast to the view held by many individuals in U. S. society.“ 
als Erst- denn als Zweitsprache gesprochen wird, und wenn es als Fremdsprache gelernt wird, dann sehr häufig als zweite nach dem Englischen (Mar-Molinero 2010: 166-167). Darin unterscheidet es sich beispielsweise vom Englischen als globale Lingua Franca. Dennoch nimmt die Anzahl der L2-Sprecher^innen des Spanischen international zu. Die Gruppe der Lernenden ist äußerst heterogen, wie Mar-Molinero (2010: 166) beobachtet:

Included in this group are those who learn Spanish as part of their educational curriculum, or in adult life, for purposes such as business or international communication. It is to this group that we should look when assessing the level of the global role of Spanish, as this is the group that represents those with a perceived need, motivation, and desire to learn Spanish voluntarily and enthusiastically.

Die Personen, die in diesem Buch nach ihrer Sichtweise auf das Spanische befragt wurden, machen einen kleinen Teil der Gemeinschaft aus, die Mar-Molinero umreißt. Kroskrity (2004: 501) weist darauf hin, dass Sprachideologien mit Interessen verknüpft sind: „[L]anguage ideologies represent the perception of language and discourse that is constructed in the interest of a specific social or cultural group.“ Welche Interessen dies bei den Spanischlernenden in Berlin sind, soll in den folgenden Kapiteln erkundet werden. Anders gesagt: Das Fallbeispiel ,Spanisch in Berlin' nimmt damit die lokalen Ausprägungen eines globalen Trends in den Blick. Die Haltungen, die in Berlin zum Spanischen geäußert werden, sind örtlich verankerte, dabei aber nicht notwendigerweise exzeptionelle Realisierungen eines weltweiten Phänomens.

Die Lernenden des Spanischen als Fremdsprache als Teil der Gemeinschaft zu sehen, die am Language Making beteiligt ist, soll auch einen kleinen Beitrag dazu leisten, den lange Zeit unhinterfragten Rahmen der Nation als definierende Einheit in der (sprach)wissenschaftlichen Praxis zu überwinden. Der national bias bzw. methodological nationalism, den Schneider (2019) identifiziert, soll damit ein Stück weit überwunden werden, ähnlich wie dies in der Forschung zum Englischen als Lingua Franca geschieht: „It studies the use of English by non-native speakers and thus does not take ethnically or nationally defined communities as starting point for research" (Schneider 2019: 2). Auch in den Untersuchungen der folgenden Kapitel stehen NichtMuttersprachler`innen im Mittelpunkt des Interesses, wobei deren nationalstaatliche oder ethnische Verortung keine übergeordnete Rolle spielt. Sie zeichnen sich vielmehr durch die Gemeinsamkeit aus, einen Lernprozess des Spanischen zu durchlaufen (oder, im Falle der Beschäftigten im Gastgewerbe, mit Spanischsprachigen häufig beruflich in Kontakt zu treten), und dies an einem gemeinsamen Ort: Berlin. Dabei steht Berlin selbst auch nicht als determinierender Rahmen der 
metasprachlichen Reflexion, sondern als beispielhaft für eine urbanisierte, globalisierte, postindustrielle europäische Gesellschaft. ${ }^{9}$

Wie Niño-Murcia, Godenzzi und Rothman (2008: 49, 54) für das Spanische als ,Weltsprache“ zeigen, gehen Globalisierung und Lokalisierung eine symbiotische Beziehung ein. Einerseits erfährt die Sprache seit der Kolonisierung eine weltweite Verbreitung, die sich mit gegenwärtiger Migration weiter fortsetzt, andererseits ergeben sich aus dieser Verbreitung wiederum neue Formen des Spanischen, die lokale Prägungen und Identifikationen widerspiegeln. Die Beispiele von Niño-Murcia et al. umfassen dabei in erster Linie solche Kontexte, in denen das Spanische als L1 stark präsent ist und mit anderen Sprachen in Kontakt tritt, etwa in den USA oder in den Grenzgebieten des südlichen Brasilien. Aber auch für Spanisch als L2 wie in Berlin kann man eine Lokalisierung des globalisierten Spanisch annehmen, insofern als die Sprache für die Lernenden ebenfalls ein Identifikationsfaktor ist: auf Basis einer bewussten Entscheidung für das Erlernen des Spanischen gewinnt man so Anbindung an die globale Sprachgemeinschaft des Spanischen, allerdings aus einem lokalen Kontext heraus, nämlich dem einer mehrsprachigen europäischen Metropole. Die Lernenden entscheiden sich, Teil einer Gemeinschaft von bis zu einer halben Milliarde Menschen zu werden, für die das Spanische in unterschiedlichster Form ein mögliches Kommunikationsmittel ist - sei es, weil sie es als Muttersprache erworben haben, sei es, weil sie es als Zweit- oder Fremdsprache in unterschiedlichem Umfang beherrschen. ${ }^{10}$

9 Vogl (2018a) zeigt, dass bei Studierenden die Faktoren Mobilität und Urbanität einen wichtigen Einfluss auf Einstellungen zu bzw. Umgang mit Mehrsprachigkeit und Sprachenlernen haben.

10 Zur Frage der Quantifizierbarkeit der spanischen Sprachgemeinschaft in der Welt vgl. MorenoFernández/Otero (2008). 
